\title{
Three-phase phase-locked loop algorithms based on sliding modes
}

\author{
Arnau Dòria-Cerezo, Víctor Repecho, and Domingo Biel
}

\begin{abstract}
This paper proposes a family of phase-locked loop schemes based on sliding modes. The use of sliding mode algorithms ensures fast response and global stability. In particular, two new algorithms are presented, both based on a complex framework for representing three-phase signals. The paper compares the obtained algorithms with the traditional schemes, and a faster response is obtained when sliding modes are used. Additionally, as an application example, the algorithm is combined with a complex-coefficient filter that allows an easy identification of both positive and negative sequence harmonics. The proposed algorithms are illustrated by numerical simulations and experimentally validated using a digital signal processor.
\end{abstract}

\section{INTRODUCTION}

\section{A. Motivation}

Phase-locked loop (PLL) algorithms are widely used for signal synchronization, and play a fundamental role in electric power applications specially in the field of grid-connected power converters. Several PLL schemes can be found in the literature for both single-phase and three-phase systems, see the extensive review papers [1] and [2], respectively. This paper is restricted to three-phase signals. Some problems with PLLs are related to: i) disturbance rejection capability, ii) improving the dynamic performance (both transient and steady-state response), and iii) reducing computational times for its implementation using low-cost industrial devices, and many papers investigate on enhancing the PLL algorithms to tackle them.

Usually, the PLL schemes have similar structures: a core algorithm aimed to obtain the phase from a relatively ideal signal, whose is used as a building block for many advanced PLLs, mainly based on a prefiltering stage to enhance the PLL capabilities. Proposing a new method for this central part of the PLLs is the aim of this paper.

Conventional PLLs are based on a synchronous reference frame PLL (SRF-PLL) scheme, that includes a phase-detector, a loop filter (usually a proportional-integral controller), and a voltage controlled oscillator, see details in [2]. Several alternatives to the SRF-PLL can be found in the literature.

This work was supported in part by the Government of Spain through the Agencia Estatal de Investigación under Project DPI2017-85404-P and in part by the Generalitat de Catalunya under Project 2017 SGR 872

A. Dòria-Cerezo is with the Dept. of Electrical Engineering and Inst of Industrial and Control Engineering, Universitat Politècnica de Catalunya, Barcelona, Spain. arnau.doria@upc.edu.

V. Repecho is with thet Inst. of Industrial and Control Engineering, Universitat Politècnica de Catalunya, Barcelona, Spain. victor.repecho. del@upc.edu.

Domingo Biel is with the Dept. of Electronic Engineering and Inst. of Industrial and Control Engineering, Universitat Politècnica de Catalunya, Barcelona, Spain. domingo.biel@upc.edu.
Type-I schemes replace the PI regulator of the SRF-PLL by a gain with a lag or lag-lead filter, resulting in a single integrator in the loop [3]. This scheme can be generalized by Type- $\mathrm{N}$ and quasi Type-N PLLs [2] (where $N$ is the number of integrators) and providing better performance, for example in presence of frequency drifts. A similar idea can be found in [4], with the use of generalized integrators. On the other hand, the enhanced-PLL (EPLL) consists of both the phase-angle and amplitude estimation loops that solve the problem related to a double-frequency ripple [5], presenting several modifications such as the pseduo-linear EPLL [6] or the generalized filtering EPLL (GEPLL) [7] [8]. Other schemes include the generalized delayed signal cancellation (GDSC)-PLL [9], moving average filters (MAF) [10], and delay-based approaches such as the adaptive cascaded delayed signal cancellation (CDSC) [11], a Type-2 PLL based on unit delays [12] or the dc-immune-PLL [13] for dc rejection.

A different approach to obtain the phase information from a signal is the use of observer-based methods. Examples include standard Luenberger observers [14], a frequency-adaptive observer [15], Kalman filters [16] [17], or sliding mode observers [18]. Finally, algebraic methods have been also proposed, see examples in [19] or [20] with the main problem of using trigonometric functions with high computational costs.

The aforementioned PLL systems can be understood as a nonlinear low pas filter (LPF). On the one hand, the presence of the LPF slows down the dynamic response of the PLL. On the other hand, the

overall scheme results in a nonlinear dynamics. In many cases, stability analyses are limited to linear approximations, see an example in [21], this issue being one of the unsolved problems when designing PLLs. See more examples in [22] or in the aforementioned paper [2]. To the authors' knowledge, nonlinear analysis of the SRF-PLL is restricted to a recent paper that considers the SRF-PLL as a high-gain observer [23], and boundedness of the trajectories can be ensured if the initial conditions are sufficiently close to the equilibrium point.

To tackle the problems related to stability and convergence time, this paper proposes the use of sliding mode methods. Sliding modes are a set of control techniques that provide fast transient responses and robustness (for example in terms of disturbance rejection) [24]. As a main drawback, these techniques require high-frequency switching actions with large gains that cause the well-known chattering phenomena. Although chattering and high frequencies could be a problem when controlling real plants, the PLL algorithm will run in a digital environment, for example a digital signal processor (DSP), where small sample time and high gains are achievable. 
The use of sliding modes for PLL has been proposed in few papers. In [25], the same scheme of a conventional SRF-PLL is used, but replacing the PI controller by sign function. Sliding modes have been also proposed in PLL schemes in [26] and [18]. In the first paper, [26], sliding modes are used together with a linear observer and finally results in a complicated algorithm for industrial applications. On the other hand, in [18], the proposed PLL is based on sliding mode observers that provide fast response, but the convergence time depends on the remaining dynamics of the observer. With respect to the above papers mentioned, the objective in this paper is to design a very simple PLLs scheme (in contrast to [26]), ensuring global stability (not proved in [25]) and with finite time convergence (faster than the asymptotic stability achieved in [18]).

Complex-valued dynamics is a useful tool for representing three-phase dynamical systems [27], because it allows reducing a third-order dynamics into a complex first-order system. This transformation is equivalent to the $\alpha \beta$ framework, commonly used in the electrical applications. Sliding modes in complex-valued systems have been proposed in [28], where it is shown that faster responses can be achieved when defining a single complex sliding manifold instead of two real sliding surfaces. Additionally, complex-coefficient filters (CCF) allow to make a distinction between positive and negative sequences when analizing unbalanced three-phase signals [29], and have been already used for harmonic selection/cancellation in SRF-PLL schemes [30]. This makes the complex-valued representation as an appropriate framework to design PLLs for unbalanced three-phase signals with high harmonic contents.

\section{B. Contributions and paper organization}

The aim of this paper is to present simple PLL schemes based on sliding modes. The main difference with respect to the PLL algorithms mentioned above is that the nonlinear LPF is replaced by a discontinuous control action to ensure sliding motion, and then global stability and fast response. In particular:

i) In comparison with other phase detector algorithms such as SRF, EPLL, Type-N or observer-based schemes, where only small-gain stability is guaranteed, the proposed PLL ensures global stability for any initial condition.

ii) Ensuring the reachability on the sliding manifold, finitetime and fast response are achieved.

iii) Contrarily to many other solutions, the voltage amplitude of the signal does not affect the stability neither the performance.

iv) Easy tuning procedure: the presented PLLs only one (or two) parameters need to be adjusted.

v) Additionally, once the sliding motion is ensured, the proposed PLL scheme does not imply any dynamics. This makes the proposed algorithm specially attractive to be combined with other dynamics such as pre-filtering stages to enhance the phase detection, or using the PLL based on sliding modes as a part of the control scheme for grid connected converters and synchronization problems.

The paper is organized as follows. After a brief introduction on the transformation from three-phase signals to complex variables in Section II, the main contribution of the paper: two PLL algorithms based on complex sliding modes, are presented in Section III. To illustrate the use of the proposed algorithms in a practical application, they are combined with a pre-filtering stage consisting in an adaptive multiple $\mathrm{CCF}$ (MCCF) in Section IV. Section V includes the experimental validation and, finally, conclusions are presented in Section VI.

\section{FROM THREE-PHASE TO COMPLEX PLANE TRANSFORMATION}

The transformation from three-phase variables to the complex plane is equivalent to the well known $a b c$ to $\alpha \beta$ transformation, see more details in [27]. The complex transformation of any periodic three-phase signal $v_{a b c}(t) \in \mathbb{R}^{3}$ consists in

$$
v(t)=T v_{a b c}(t)
$$

where $v(t) \in \mathbb{C}$ is the complex signal, and $T \in \mathbb{C}^{3}$ is the complex vector

$$
T=c\left(1,-\frac{1}{2}+j \frac{\sqrt{3}}{2},-\frac{1}{2}-j \frac{\sqrt{3}}{2}\right),
$$

where $c=2 / 3$ to preserve the amplitude in the complex transformation on balanced three-phase voltages. Then, $v(t)$ results in a periodic orbit on the complex plane.

Transformation (1) is not bijective, and the reconstruction of $v_{a b c}(t)$ from $v(t)$ requires more information. In electrical three-phase systems, this information is the homopolar component, i.e., the sum of all the components of $v_{a b c}(t)$,

$$
v_{0}(t)=v_{a}(t)+v_{b}(t)+v_{c}(t),
$$

where $v_{0}(t) \in \mathbb{R}$.

If $v_{a b c}$ is a set of balanced three-phase voltages

$$
v_{a b c}=V\left(\begin{array}{c}
\cos \left(\omega_{o} t+\phi\right) \\
\cos \left(\omega_{o} t-\frac{2 \pi}{3}+\phi\right) \\
\cos \left(\omega_{o} t+\frac{2 \pi}{3}+\phi\right)
\end{array}\right),
$$

where $V$ is the amplitude and $\omega_{o}$ is the frequency, the complex voltage obtained from transformation (1) is

$$
v=V e^{j \theta}
$$

where $\theta=\omega_{o} t+\phi$ is the phase of the three-phase signal.

\section{Phase-LOCKed LOOP ALGORITHMS BASED ON SLIDING MODES}

In this section, two sliding mode-based PLLs are presented. Both algorithms follow the scheme shown in Figure 1 and are based on the complex switching function defined by

$$
\sigma=\hat{V}-v e^{-j \hat{\theta}}
$$

where $\hat{V}, \hat{\theta} \in \mathbb{R}$ are the estimation of the voltage amplitude and phase of (2). The real and imaginary parts of (3) can be written separately as

$$
\begin{aligned}
\sigma_{R} & =\hat{V}-V \cos (\theta-\hat{\theta}) \\
\sigma_{I} & =-V \sin (\theta-\hat{\theta})
\end{aligned}
$$




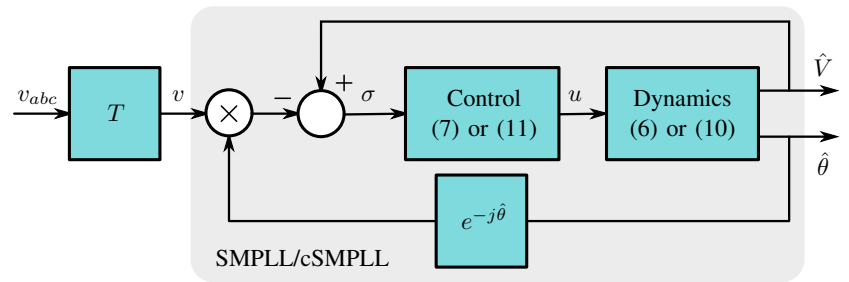

Fig. 1: Scheme of the sliding mode-based PLL (SMPLL and cSMPLL).

Notice that

$$
\begin{aligned}
\hat{\theta} & =\theta \\
\hat{V} & =V,
\end{aligned}
$$

is a solution of $\sigma=\sigma_{R}+j \sigma_{I}=0$.

\section{A. The PLL based on sliding modes (SMPLL)}

Based on (5), the next step is to propose a control system where the control goal is to reach (and remain) in $\sigma=0$, see Figure 1. For simplicity, the plant dynamics is chosen as a pure complex integrator of the complex control input $u=u_{R}+j u_{I}$, where the outputs correspond to the estimated amplitude and phase, respectively, i.e.,

$$
\begin{gathered}
\dot{\hat{V}}=u_{R} \\
\dot{\hat{\theta}}=u_{I} .
\end{gathered}
$$

According to the dynamics in (6) and the complex switching function (3) the following control law is designed to independently reach $\sigma_{R}=0$ and $\sigma_{I}=0$,

$$
\begin{gathered}
u_{R}=-k_{R} \operatorname{sign}\left(\sigma_{R}\right) \\
u_{I}=-k_{I} \operatorname{sign}\left(\sigma_{I}\right) .
\end{gathered}
$$

Proposition 1: The Sliding Mode Phase-Locked Loop (SMPLL) in Figure 1 with the dynamics and control functions defined in (6) and (7), respectively, guarantees the convergence of $(\hat{\theta}, \hat{V})$ to $(\theta, V)$ if conditions

$$
\begin{aligned}
& k_{R}>0 \\
& k_{I}>|\omega|
\end{aligned}
$$

are fulfilled.

Proof. The proof has two parts:

Part I: Sliding motion on the imaginary axis: Let us split the switching function $\sigma_{I}$ in three regions

$$
\begin{array}{ll}
\text { Region A : } & (4 n-1) \frac{\pi}{2}<\theta-\hat{\theta}<(4 n+1) \frac{\pi}{2} \\
\text { Region B : } \quad(2 n-1) \pi<\theta-\hat{\theta}<(4 n-1) \frac{\pi}{2} \\
\text { Region C : } \quad(4 n+1) \frac{\pi}{2}<\theta-\hat{\theta}<(2 n+1) \pi
\end{array}
$$

where $n \in \mathbb{Z}$. See, in Figure 2, the defined regions for $n=0$.

- Sliding modes in Region A: Differentiating (4b) and using (6b)-(7b) one have

$$
\dot{\sigma}_{I} \sigma_{I}=-\sigma_{I} V \cos (\theta-\hat{\theta})\left(\omega+k_{I} \operatorname{sign}\left(\sigma_{I}\right)\right) .
$$

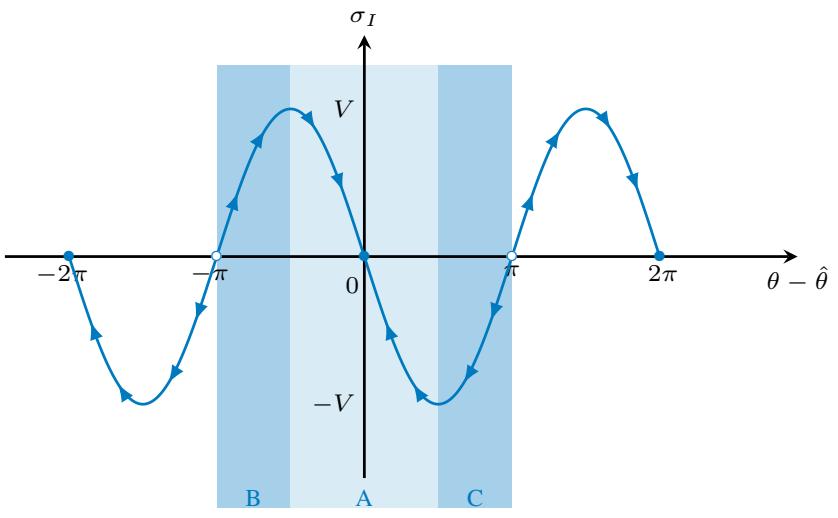

Fig. 2: Phase portrait for $\sigma_{I}$ with regions $\mathrm{A}, \mathrm{B}$ and $\mathrm{C}$ for $n=0$. Dotted and circled points on $\sigma_{I}=0$ correspond to attractive and repulsive points, respectively. Arrows in $\sigma_{I}$ indicate the trajectories.

Since $V>0$, with (8b) and (9a) one gets $\sigma_{I} \dot{\sigma}_{I}<0$ and sliding modes on

$$
\theta-\hat{\theta}=2 n \pi,
$$

or, equivalently (5a), are guaranteed.

- Moving from Region B to Region A: Dynamics in (6b) with (7b) in the interval defined by (9b) results in $\dot{\hat{\theta}}=$ $-k_{I}$, and the rate

$$
\dot{\theta}-\dot{\hat{\theta}}=\omega+k_{I}>0
$$

where (8b) is used. Then, trajectories in Region B move to Region A.

- Moving from Region C to Region A: Similarly, in the interval defined by (9c), trajectories in Region $\mathrm{C}$ move to Region A.

Figure 2 summarises this part of the proof.

Part II: Sliding motion on the real axis: Differentiating (4a) and using (6) yields

$$
\sigma_{R} \dot{\sigma}_{R}=\left(u_{R}+V\left(\omega-u_{I}\right) \sin (\theta-\hat{\theta})\right) \sigma_{R}
$$

Since sliding motion on the imaginary axis has been proved, $u_{I}$ takes the equivalent control value, $u_{I, e q}=\omega$, and $\theta-\hat{\theta}=$ $2 n \pi$. Then, using (7a), the latter simplifies into

$$
\dot{\sigma}_{R} \sigma_{R}=-k_{R} \sigma_{R} \operatorname{sign}\left(\sigma_{R}\right)<0,
$$

and sliding modes on $\sigma_{R}=0$ are guaranteed since $\sigma_{R} \dot{\sigma}_{R}<0$, if (8a) is verified.

\section{B. The PLL based on complex sliding modes (cSMPLL)}

Alternatively, another design can be obtained based on the complex sliding modes proposed in [28]. Using the scheme in Figure 1, let us consider the complex-valued dynamics

$$
\dot{\hat{v}}=u e^{j \hat{\theta}}
$$

where $\hat{v}=\hat{V} e^{j \hat{\theta}}$ is the estimated complex voltage, and let us define the complex switching action as

$$
u=-k \frac{\sigma}{|\sigma|},
$$


where $\sigma$ is the complex switching function defined in (3), and the real gain $k$ fulfills

$$
k-|\omega| V>\epsilon>0 .
$$

Notice that the dynamics in (10) is equivalent to

$$
\dot{\hat{V}}+j \dot{\hat{\theta}} \hat{V}=u,
$$

that, using $u=u_{R}+j u_{I}$, can be split in the real and imaginary dynamics

$$
\begin{aligned}
\dot{\hat{V}} & =u_{R} \\
\dot{\hat{\theta}} & =\frac{1}{\hat{V}} u_{I} .
\end{aligned}
$$

Then (13) is similar to (6), but dividing by $\hat{V}$ in (13b). This reminds of the amplitude normalization scheme (ANS) often proposed for the SRF-PLL [31].

Proposition 2: The complex Sliding Mode Phase-Locked Loop (cSMPLL) in Figure 1 with the dynamics and control functions defined in (10) and (11), respectively, guarantees the convergence of $(\hat{\theta}, \hat{V})$ to $(\theta, V)$ if condition (12) is fulfilled. Additionally, the convergence to (5) is guaranteed in finite time, and the reaching time is bounded by

$$
T=\frac{\sqrt{2}}{\epsilon} \sqrt{W\left(t_{0}\right)}
$$

where $t_{0}$ is the initial time.

Proof. As suggested in [28], let use the Lyapunov function candidate $^{1}$

$$
W=\frac{1}{2} \sigma^{*} \sigma .
$$

Calculating its time derivative and using the complex switching function (3) and the dynamics defined in (10) one have

$$
\dot{W}=\operatorname{Re}\left(\sigma^{*} \dot{\sigma}\right)=\operatorname{Re}\left(\sigma^{*}\left(\dot{\hat{V}}-j(\omega-\dot{\hat{\theta}}) V e^{j(\theta-\hat{\theta})}\right)\right)
$$

Then, using (3),

$$
\begin{aligned}
\dot{W} & =\operatorname{Re}\left(\sigma^{*}\left(u_{R}+j \frac{u_{I}}{\hat{V}}(\hat{V}-\sigma)-j \omega V e^{j(\theta-\hat{\theta})}\right)\right) \\
& =\operatorname{Re}\left(\sigma^{*}\left(u-j \frac{u_{I}}{\hat{V}} \sigma-j \omega V e^{j(\theta-\hat{\theta})}\right)\right) \\
& =\operatorname{Re}\left(-j \frac{u_{I}}{\hat{V}}|\sigma|^{2}+\sigma^{*}\left(u-j \omega V e^{j(\theta-\hat{\theta})}\right)\right) \\
& =\operatorname{Re}\left(\sigma^{*}\left(u-j \omega V e^{j(\theta-\hat{\theta})}\right)\right),
\end{aligned}
$$

and, finally, with (11) and denoting the argument of $\sigma$ as $\phi$ one gets

$$
\begin{aligned}
\dot{W} & =\operatorname{Re}\left(\sigma^{*}\left(-k \frac{\sigma}{|\sigma|}-j \omega V e^{j(\theta-\hat{\theta})}\right)\right) \\
& =|\sigma| \operatorname{Re}\left(-k-j \omega V e^{j(\theta-\hat{\theta}-\phi)}\right) \\
& =-|\sigma| \operatorname{Re}(k-\omega V \sin (\theta-\hat{\theta}-\phi)) \\
& \leq-(k-\omega V)|\sigma|,
\end{aligned}
$$

that ensures $\dot{W}<0$ by condition (12), and convergence to $\sigma=0$ is guaranteed.

\footnotetext{
${ }^{1}(\cdot)^{*}$ denotes the complex conjugate of $(\cdot)$.
}
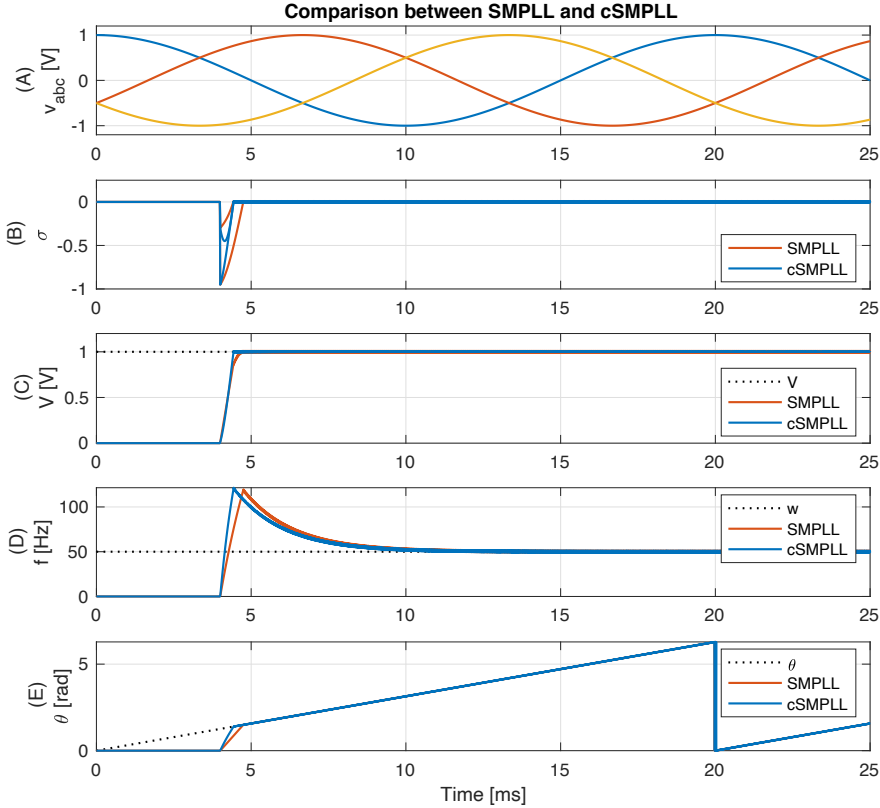

Fig. 3: Simulation results: comparison between the SMPLL and the complex approach, cSMPLL. (A) Three-phase voltage signal, $v_{a b c}$, (B) Real and imaginary parts of the complex switching function, $\sigma,(\mathrm{C})$ Estimated voltage amplitude, $\hat{V}$, (D) Estimated voltage frequency $\hat{f}=\frac{\hat{\omega}}{2 \pi}$, (E) Estimated voltage phase $\hat{\theta}$. In plots $\mathrm{B}, \mathrm{C}, \mathrm{D}$ and $\mathrm{E}$, red corresponds to the SMPLL and blue to cSMPLL.

The finite time convergence is proved as follows. From (15) with (12) we can write

$$
\begin{aligned}
\dot{W} & <-\epsilon|\sigma| \\
& =-\sqrt{2} \epsilon W^{\frac{1}{2}}
\end{aligned}
$$

and it follows that $W(t)=0$ for $t-t_{0}>T$, with (14).

\section{Comparison between the two SMPLL approaches}

The SMPLL algorithm shown in Figure 1 admits two alternatives: the one in Section III-A, defining separately two sliding surfaces which results in using (6) and (7), or the complex approach obtained in Section III-B that results in (10) and (11). Both algorithms are compared, in simulations using Matlab/Simulink, with a $1 \mathrm{~V} 50 \mathrm{~Hz}$ three-phase voltage, the initial conditions of the PLL algorithms being $\hat{\theta}(0)=0$, $\hat{V}(0)=0.01 \mathrm{~V}$, and with the gains have been set to $k_{R}=$ $k_{I}=3 \cdot 10^{3}$ and $k=3 \sqrt{2} \cdot 10^{3}$. Note that $k_{R}, k_{I}$ and $k$ are chosen such that the control law (11) generalises (7), see details in [28]. Equations (7) are approximated by a hysteresis function with a band of $\epsilon=10^{-2}$, and (11) is implemented with a complex hysteresis with a radius of $\epsilon=10^{-2}$ (see again [28]). Finally, the estimated voltage frequency $\hat{\omega}$ is obtained by passing $\dot{\hat{\theta}}$ through a first order filter with a cut-off frequency of $100 \mathrm{~Hz}$.

Figure 3 shows the response of the PLLs based on sliding modes. In both cases, the phase and amplitude are reached after a short transient time, around $0.5 \mathrm{~ms}$. Notice that, differently from the SMPLL, when the cSMPLL is used the real and imaginary components of $\sigma=0$ are reached at the same time, resulting in a faster response. See details of 

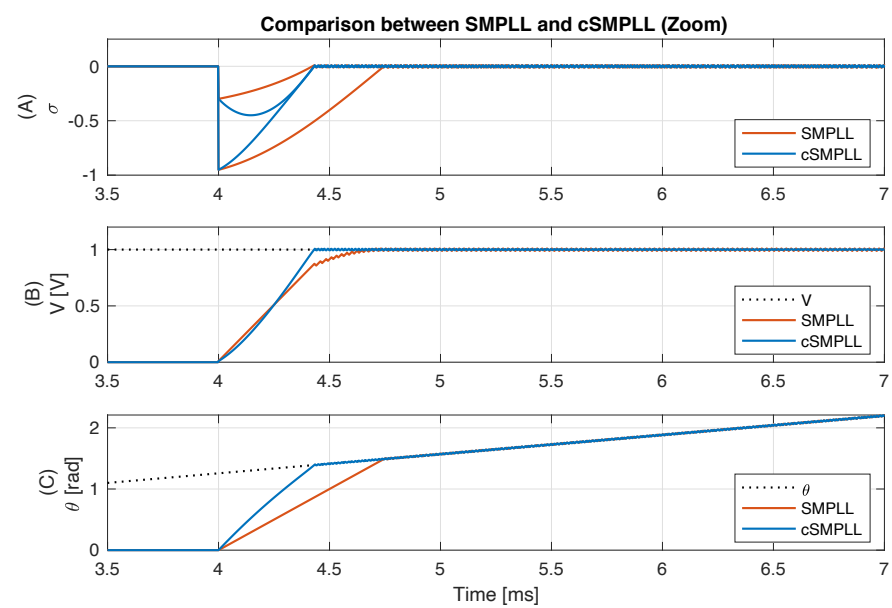

Fig. 4: Simulation results: zoom of the comparison between the SMPLL and cSMPLL algorithms. (A) Real and imaginary parts of the complex switching function, $\sigma$, (B) Estimated voltage amplitude, $\hat{V}$, (C) Estimated voltage phase $\hat{\theta}$. Red plots correspond to the SMPLL and blue plots to cSMPLL.

the transient response in Figure 4. Also, thanks to the small hysteresis band, chattering phenomena can be neglected.

The obtained responses are very close. The main differences are:

i) with the same gains the cSMPLL is faster than the SMPLL, but

ii) the cSMPLL dynamics is more complicated and, as pointed out when written as in (13), has an implicit additional division, see differences between (6b) and (13b).

\section{APPLICATION EXAMPLE: CSMPLL WITH COMPLEX COEFFICIENT FILTERS}

In the previous Section, the two PLL algorithms based on sliding modes were presented. It was shown that the cSMPLL exhibits a better behaviour with a faster response. In this Section, to show the applicability of the algorithm, the cSMPLL is merged with complex adaptive filters in order to improve the performance in case of distorted signals.

\section{A. $C S M P L L-C C F$}

Filter capabilities are commonly needed when designing phase detector algorithms. Several approaches are proposed to remove these components, such as moving average filters, Notch filters, delayed signal cancellation, or second-order generalized integrators (SOGI) algorithms, see an extensive comparison among different methods in [22]. One alternative is the use of CCFs before the PLL input. The main feature of the CCFs is that they directly distinguish between positive and negative sequences [29] which make them specially interesting for unbalanced signals. The transfer function of a first order $\mathrm{CCF}$ is

$$
G_{\mathrm{CCF}}(s)=\frac{\gamma}{s-j \omega_{1}+\gamma}
$$

where $\omega_{1}$ is the fundamental frequency and $\gamma$ is the cutoff frequency that defines the bandwidth of the filter.

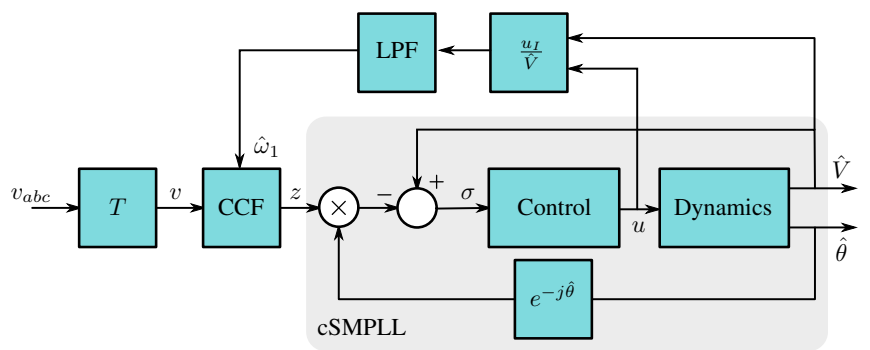

Fig. 5: Scheme of the cSMPLL with a CCF.

The differential form of (16) is

$$
\dot{z}=\left(j \omega_{1}-\gamma\right) z+\gamma v .
$$

In many applications the frequency is varying and the CCF needs to be adapted to the new frequency values. To this end, an adaptive version of the cSMPLL-CCF scheme is proposed in Figure 5, where the value of the estimated frequency is obtained by using a LPF for the $\frac{u_{I}}{\hat{V}}$ signal.

Using the frequency estimation of cSMPLL in (17), the dynamics can be written as

$$
\begin{aligned}
\dot{z} & =\left(j \hat{\omega}_{1}-\gamma\right) z+\gamma v \\
\tau \dot{\hat{\omega}}_{1} & =-\hat{\omega}_{1}+\frac{1}{\hat{V}} u_{I}
\end{aligned}
$$

where $\tau$ is the inverse of the cut-off frequency of the LPF. If the SMPLL is used instead of cSMPLL (18b) becomes

$$
\tau \dot{\hat{\omega}}_{1}=-\hat{\omega}_{1}+u_{I} .
$$

Figure 6 shows the comparison between the cSMPLL and the cSMPLL-CCF. For this simulation test, the three-phase voltage is $1 \mathrm{~V} 50 \mathrm{~Hz}$ containing white noise. The cSMPLL parameters are those of the simulations in Section III, the LPF has a cut-off frequency of $100 \mathrm{~Hz}$ and the damping ratio of the CCF is set to 0.707 (which is shown to be the optimal value [29]), that results in $\gamma=0.707 \cdot 2 \pi 50$. During this test, after $10 \mathrm{~ms}$ the frequency suddenly changes up to $75 \mathrm{~Hz}$. Notice that, thanks to the adaptive law, the CCF rejects the noise and the estimated values from the cSMPLL-CCF are less noisy than the obtained directly from the cSMPLL. As a counter-part, the response becomes slower because the CCF dynamics. Thanks to the quasi-static behaviour of the cSMPLL (due to the fast response), the error behaviour for cSMPLLCCF in Figure 6(D) coincides with the linearized response from (18), thus revealing that for stability analysis in other closed-loop applications, the SMPLLs can be considered as static functions.

The merge of the adaptive CCF with the CSMPLL can be used to illustrate the aforementioned stability properties of the sliding mode PLLs with respect to an standard method such as the SRF-PLL. The comparison is done in a simulation environtment, using the same signal before, a $1 \mathrm{~V} 50 \mathrm{~Hz}$ three phase-voltage, and consists in the following steps:

i) The first step is aimed to adjust the gains of the two strategies (without the CCF) to have the same settling time for the worst initial conditions, i.e., $\theta(0)=\frac{\pi}{2} \mathrm{rad}$. The obtained gains are $k=3 \sqrt{2} \cdot 10^{3}$, for the cSMPLL, 

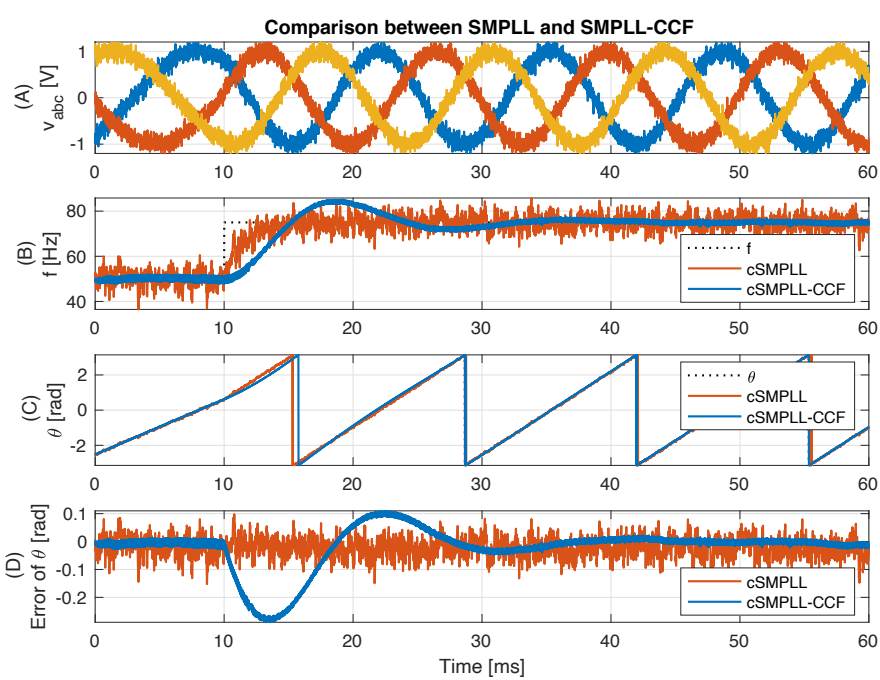

Fig. 6: Simulation results: comparison between the cSMPLL and cSMPLLCCF approaches. (A) Three-phase voltage signal, $v_{a b c}$, (B) estimated voltage frequency $\hat{f}=\frac{\hat{\omega}}{2 \pi}$, (C) estimated voltage phase $\hat{\theta}$, and (D) error of the estimated voltage phase.

and $k_{p}=8000, k_{i}=3.5 \cdot 10^{6}$, for the SRF-PLL. The resulting dynamics is shown in Figure 7(A), and zoomed in Figure 7(B). Notice that the phase in both schemes reaches its actual value in approximately the same time, around $0.5 \mathrm{~ms}$. Roughly speaking, the two strategies are equally adjusted and ready for a fair comparison

ii) The second step consists in merging the adaptive CCF, as shown in Figure 5 (and equivalently for the SRF-PLL). Notice that the estimation of the frequency, $\hat{\omega}$, is now feedback to the adaptive CCF, thus the dynamics of the PLL is now affecting the CCF dynamics. The obtained behaviours are shown in Figure 7(C). The response with the SRF-PLL turns to be unstable (in red), but the response of the cSMPLL remains stable (in blue). This behaviour is thanks to the global stability proof and the sliding motion, that ensures the cSMPLL behaving as a quasi static element.

iii) A third step can be done by searching the SRF-PLL gains that ensure stability when combined with the adaptive CCF. These values are found at $k_{p}=800$ and $k_{i}=$ $1.765 \cdot 10^{5}$ (the ones used in Section V-A). Figure 7(D) shows that the response of the scheme with SRF-PLL (red) is now stable but is much oscillating and slower than the one obtained with the CSMPLL.

Then we can conclude that, at equal conditions, the cSMPLL improves the behaviour with respect to the SRF-PLL.

\section{B. $C S M P L L-M C C F$}

When three-phase signals content higher harmonics the performance of the PLL decays. Reducing the loop bandwidth of the PLL alleviates the harmonic content, but with the cost of increasing the transient settling time. An alternative is to remove other frequencies than the fundamental one. The MCCF is composed by a set of CCF modules that extracts information of the harmonic components [29] and allows selective harmonic cancellation [30]. The scheme of the
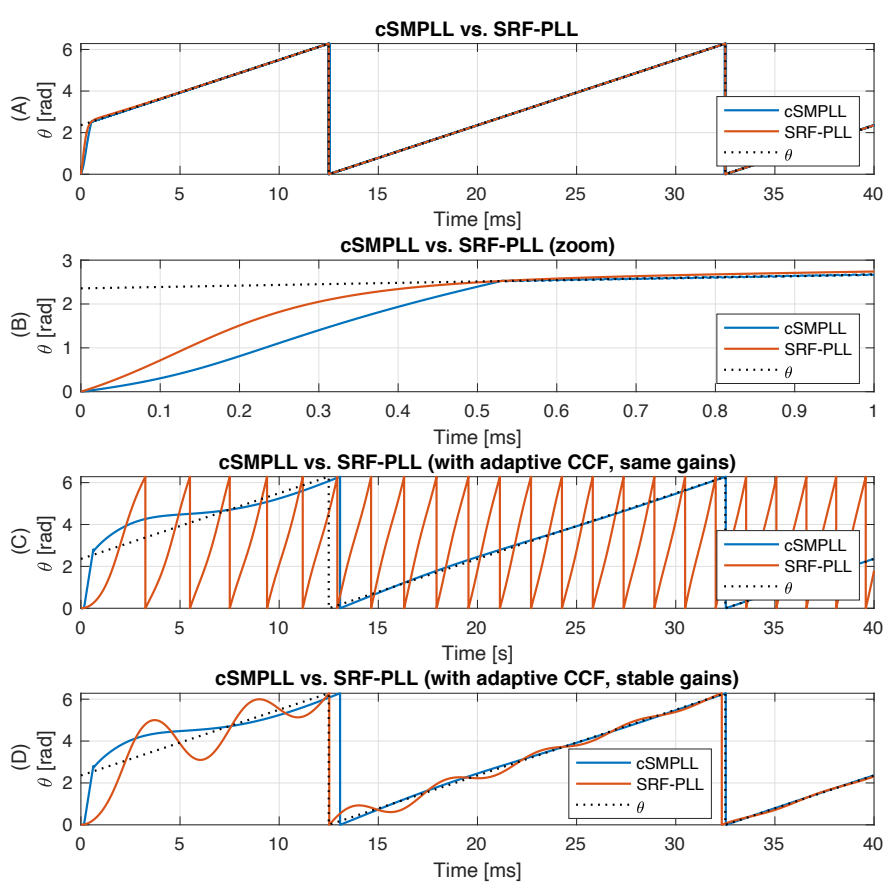

Fig. 7: Simulation results: comparison between the cSMPLL and the SRFPLL.

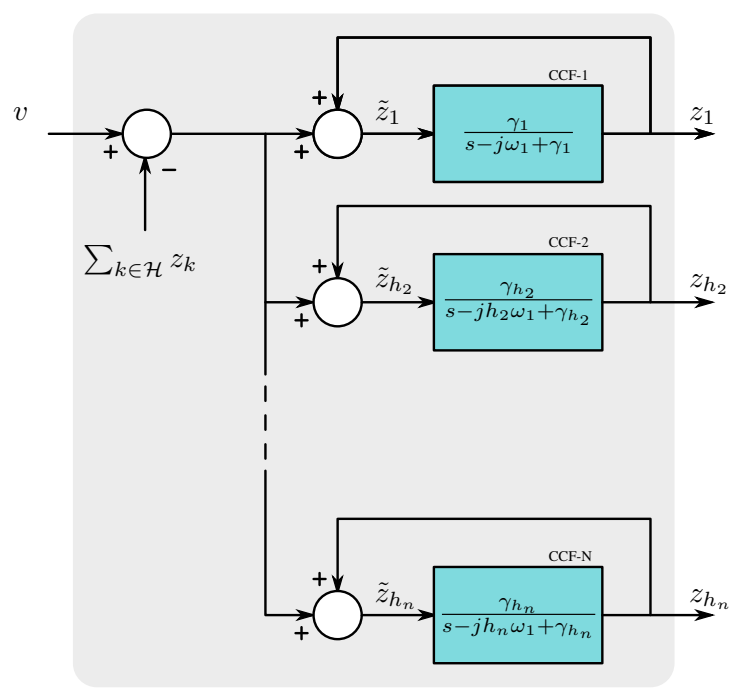

Fig. 8: Scheme of the MCCF.

MCCF is depicted in Figure 8, where $\omega_{1}$ is the fundamental angular frequency, $\mathcal{H}=\left\{1, h_{2}, \ldots, h_{n}\right\} \in \mathbb{Z}^{n}$ is the set of harmonics considered in the filter. The stability for any positive $\gamma_{k}$ values is proved in [32].

The MCCF algorithm is connected to the cSMPLL as shown in Figure 9. Optionally, if one wants to extract phase and amplitude for each harmonic, additional cSMPLL blocks could be included. In the case of unknown or varying frequency, the value of $\omega_{1}$ should be fed back from cSMPLL-1 by means of a LPF.

The proposed cSMPLL-MCCF algorithm is tested as an harmonic extractor using the full scheme proposed in Figure 9 with a cSMPLL for each harmonic. This test has been numerically performed to easily view all the reconstructed signals. The tests consists on a three phase voltage signal 


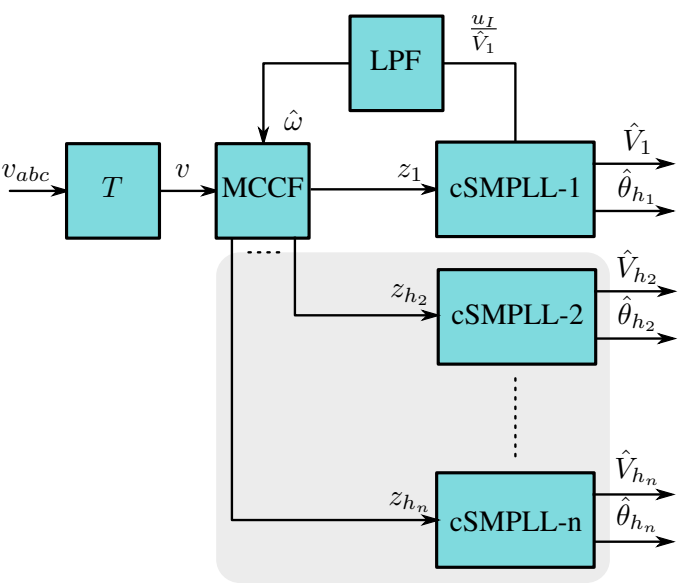

Fig. 9: Scheme of the cSMPLL with the MCCF. Blocks in the shadowed area are optional for extracting information of harmonic components.
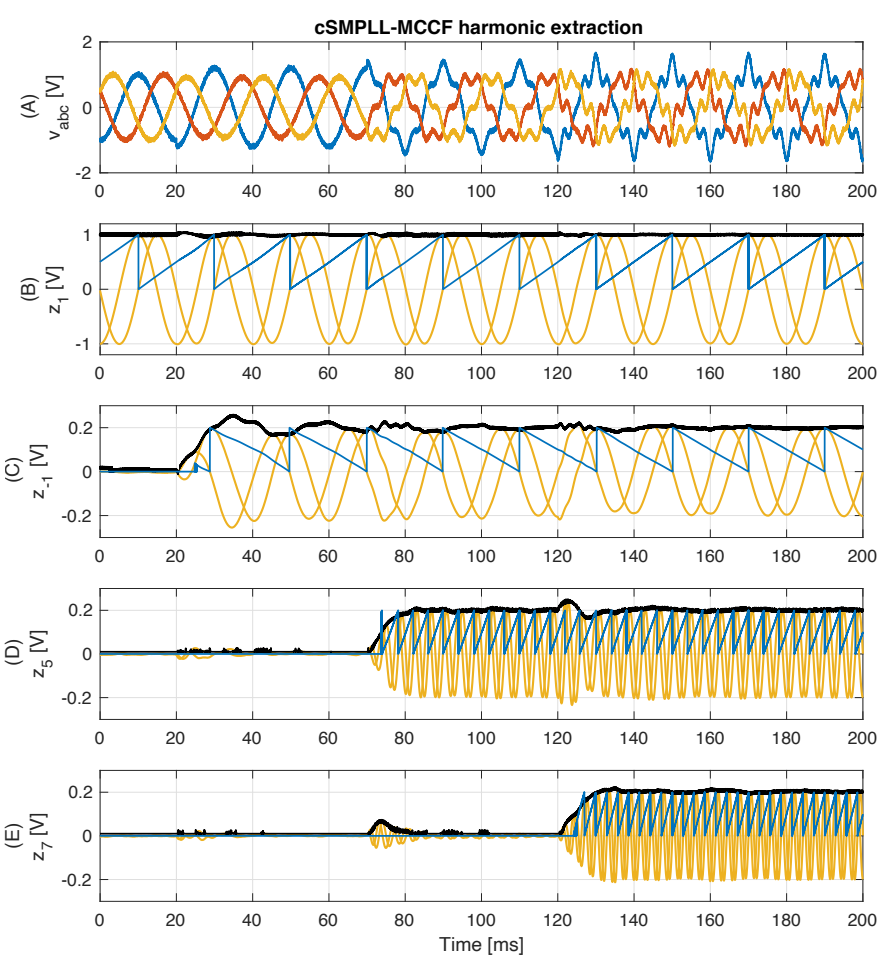

Fig. 10: Simulation results: the cSMPLL-MCCF algorithm as an harmonic extractor. (A) Three-phase voltage signal, $v_{a b c}(t),(\mathrm{B}, \mathrm{C}, \mathrm{D}, \mathrm{E})$ Complex harmonic voltages $\left(z_{1}, z_{-1}, z_{5}, z_{7}\right)$ obtained from the MCCF (in yellow), with the amplitudes (in black) and phases (in blue) generated by each cSMPLL. For an easy visualization, phase signals are rescaled with the amplitude of each harmonic.

$1 \mathrm{~V} 50 \mathrm{~Hz}$, and three harmonics components are included: negative sequence, fifth harmonic and seventh harmonic, all with $0.2 \mathrm{~V}$ amplitude at $t=20 \mathrm{~ms}, t=70 \mathrm{~ms}$ and $t=120 \mathrm{~ms}$, respectively. Figure 10 shows the three-phase voltages, and the reconstruction of each complex (or $\alpha \beta$ ) voltages, the phase and the amplitude corresponding to each harmonic. After each change, the algorithm is able to extract the information of the harmonics in, approximately, $20 \mathrm{~ms}$.

\section{EXPERIMENTAL VALIDATION}

The presented schemes have been digitally implemented in a Hardware-in-the-Loop platform using the Typhoon HIL402

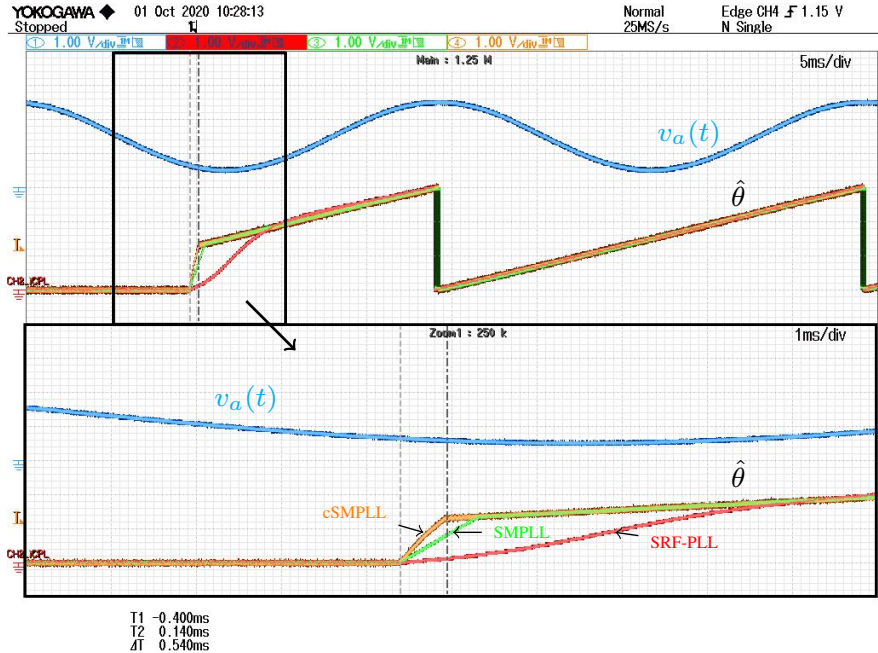

Fig. 11: Experimental results: comparison of the starting behaviour. (top) $v_{a}(t)$ voltage $(\mathrm{CH} 1$, blue), (bottom) estimated phase $\hat{\theta}$ : SRF-PLL $(\mathrm{CH} 2$, red), SMPLL (CH3, green) and cSMPLL (CH4, orange).

device and the F28379 micro-controller from Texas Instruments, often used for the control of power converters. The HIL platform has been used to generate the three phase signals with the corresponding voltage sags, phase jumps and the harmonic content. All the algorithms have been programmed using the Matlab/Simulink code generation tool configured to optimize the execution time in the F28379. The sampling frequency executing the algorithms has been set to $200 \mathrm{kHz}$. All signals have been measured using the DAC channels from the micro-controller. Two tests are proposed to validate the implementation in a micro-controller: the first test consists in the comparison of the sliding mode PLLs with respect to a standard PLL, such as the SRF-PLL, while the second test propose testing the cSMPLL-MCCF scheme.

\section{A. Test 1: sliding mode PLLs vs SRF-PLL}

The SMPLL and cSMPLL algorithms shown in Figure 1 with (7) and (11), respectively, are compared with the standard SRF-PLL algorithm. The three-phase signals are $1 \mathrm{~V}$ amplitude and $50 \mathrm{~Hz}$ as fundamental frequency. The gains of the PLLs based on sliding modes are those used in Section III-C. The PI gains of the SRF-PLL have been set to $k_{p}=800$ and $k_{i}=1.765 \cdot 10^{5}$ to achieve a fast response but ensuring stability when the PLL is combined with the filter stage proposed in Section IV. See more details about the gains adjustment of the SRF-PLL in Section IV-A.

Figure 11 shows the comparison when starting the SMPLL, cSMPLL and SRF-PLL algorithms. As expected, the phase synchronization of SMPLL and cSMPLL algorithms are similar to the ones obtained in simulations, and faster in comparison to the SRF-PLL (the red signal). In particular, with the cSMPLL the actual phase value is reached in $0.54 \mathrm{~ms}$, the SMPLL requires $0.72 \mathrm{~ms}$, and the SRF-PLL takes more than $5 \mathrm{~ms}$.

Figure 12 shows the phase portrait of the real and imaginary parts of $\sigma$ during the starting behaviour. It can be observed that, when using the SMPLL and the cSMPLL, the switching 


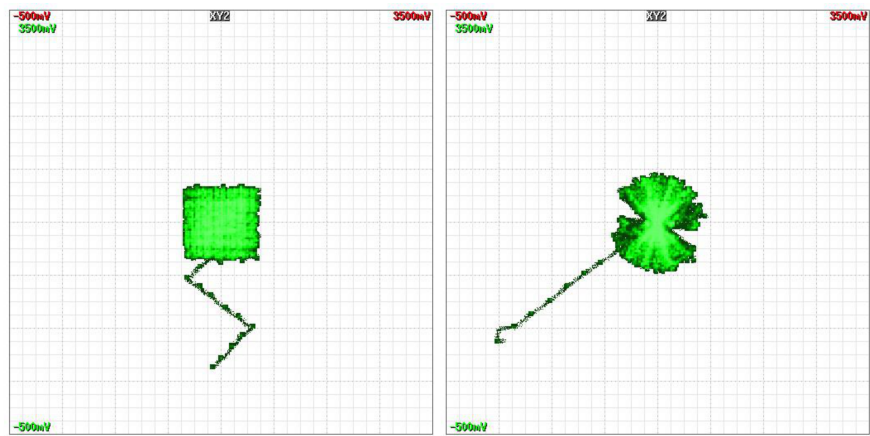

Fig. 12: Experimental results: $x y$ plot from the oscilloscope to represent the phase portrait of $\sigma_{R}, \sigma_{I}$ for the SMPLL (left) and CSMPLL (right).

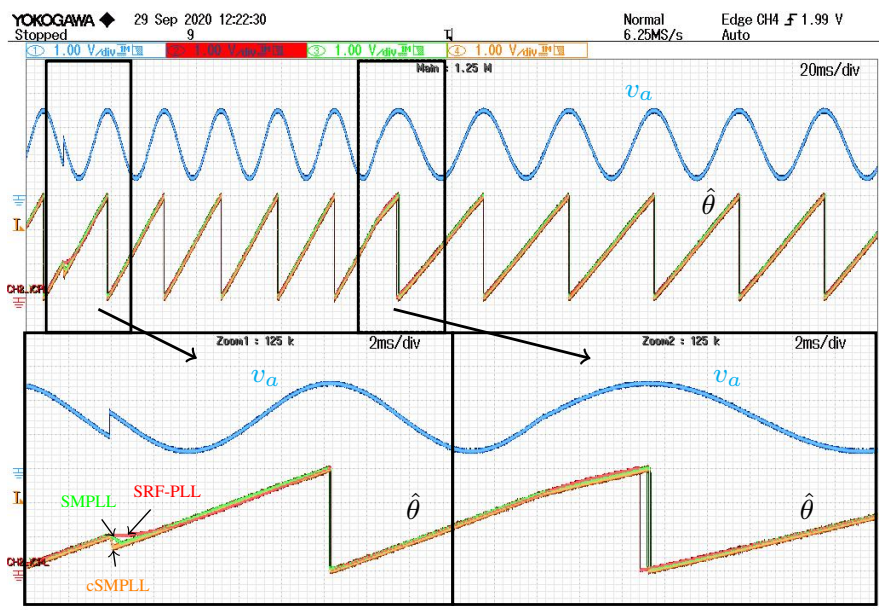

Fig. 13: Experimental results: comparison in the face of a phase jump lagging $45^{\circ}$ and, $60 \mathrm{~ms}$ later, a change of frequency from $75 \mathrm{~Hz}$ to $50 \mathrm{~Hz}$. SRF-PLL $(\mathrm{CH} 2$, red), SMPLL (CH3, green) and cSMPLL (CH4, orange).

function remains in the square defined by the hysteresis band, $\epsilon$, (see the left plot) or in the ball of radius $\epsilon$, (right plot) respectively. See [28] for a further discussion about these phenomena.

In the experiment shown in Figure 13 the three algorithms (SMPLL, cSMPLL and SRF-PLL) are compared in the face of a phase jump lagging $45^{\circ}$ and, $60 \mathrm{~ms}$ later, a change of frequency from $75 \mathrm{~Hz}$ to $50 \mathrm{~Hz}$. Similarly to the starting behaviour, both the SMPLL and the cSMPLL are considerably faster than the conventional SRF-PLL, specially when a phase jump occurs.

The execution time with the used micro-controller is the same for both SMPLL and cSMPLL schemes: $890 \mathrm{~ns}$. This corresponds to $17.8 \%$ of the sampling time and the remaining time is available for including additional features to the PLL, such as the filtering stage proposed in Section IV.

\section{B. Test 2: cSMPLL-MCCF validation}

The cSMPLL-MCCF scheme shown in Figure 9 has been implemented in the micro-controller. The three-phase signal is now $1 \mathrm{~V} 50 \mathrm{~Hz}$ including the 5th and 7th harmonic components (with $0.1 \mathrm{~V}$ amplitude each). Four tests have been carried out with the cSMPLL-MCCF algorithm: harmonic

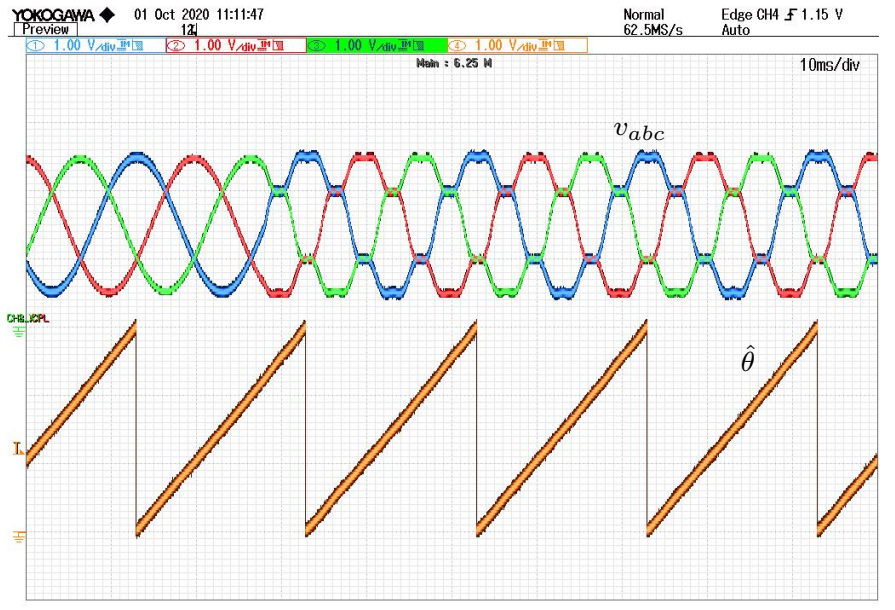

Fig. 14: Experimental results: behaviour of the cSMPLL-MCCF when the three-phase voltage is affected by the 5 th and the 7 th harmonic (with $0.1 \mathrm{~V}$ amplitude each). Three-phase voltages, $v_{a b c}(t),(\mathrm{CH} 1, \mathrm{CH} 2, \mathrm{CH} 3)$ and the estimated phase $\hat{\theta}$ ( $\mathrm{CH} 4$, orange).

response, a phase jump, a frequency change and a partial voltage sag.

Figure 14 shows the behaviour of the algorithm when the harmonics suddenly appear in the three-phase voltage. Thanks to the combination of the CSMPLL with the MCCF, the phase remains tracked by the PLL scheme without oscillations due to the harmonics.

Figure 15 shows the response of the algorithm when the phase jumps $45^{\circ}$ (lagging and then leading). The estimated phase is recovered in less than one cycle. With respect to the previous experiments in Section V-A, the phase estimation became slower because the dynamics of the MCCF. Also, in steady state, the harmonic content is rejected by the MCCF and the phase estimation does not oscillate.

The response in the face of a frequency change is shown in Figure 16. Similarly to the previous phase shift case, the algorithm smoothly recovers the actual phase value in less than one cycle. In Figure 17, the response when an unbalanced sag occurs is shown with a fast response, around half cycle. Notice that negative sequence harmonics appear because unbalanced voltages are almost instantaneously rejected by the MCCF.

The execution time of the overall cSMPLL-MCCF algorithm is $1255 \mathrm{~ns}$, that corresponds to $25.1 \%$ of the sampling time when working at $200 \mathrm{kHz}$.

\section{Conclusions}

In this paper, two PLL algorithms based on sliding modes have been proposed. The main features of the presented scheme are:

i) Global stability: despite other PLL algorithms, the proposed methods are globally stable. This allows to set any initial condition and high gains without stability problems.

ii) Finite time convergence: the SMPLL and the CSMPLL algorithms exhibit finite time convergence, instead of the asymptotic one obtained in the traditional algorithms. 


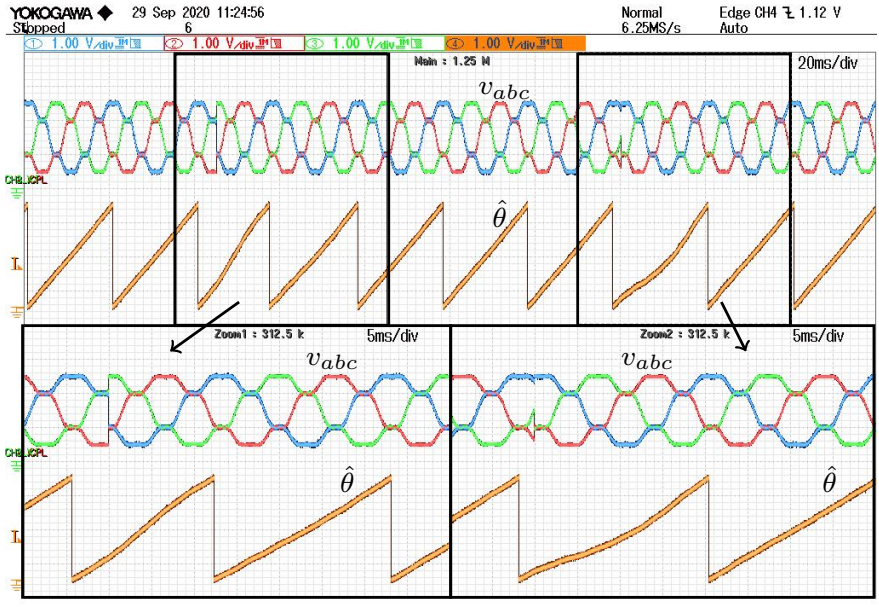

Fig. 15: Experimental results: behaviour of the CSMPLL-MCCF in the face of a phase jump, lagging $45^{\circ}$ and, after $60 \mathrm{~ms}$, leading $45^{\circ}$. Three-phase voltages, $v_{a b c}(t),(\mathrm{CH} 1, \mathrm{CH} 2, \mathrm{CH} 3)$ and the estimated phase $\hat{\theta}(\mathrm{CH} 4$, orange). Zoom 1 (bottom left): behaviour when the phase lags $45^{\circ}$. Zoom 2 (bottom right): behaviour when the phase leads $45^{\circ}$.

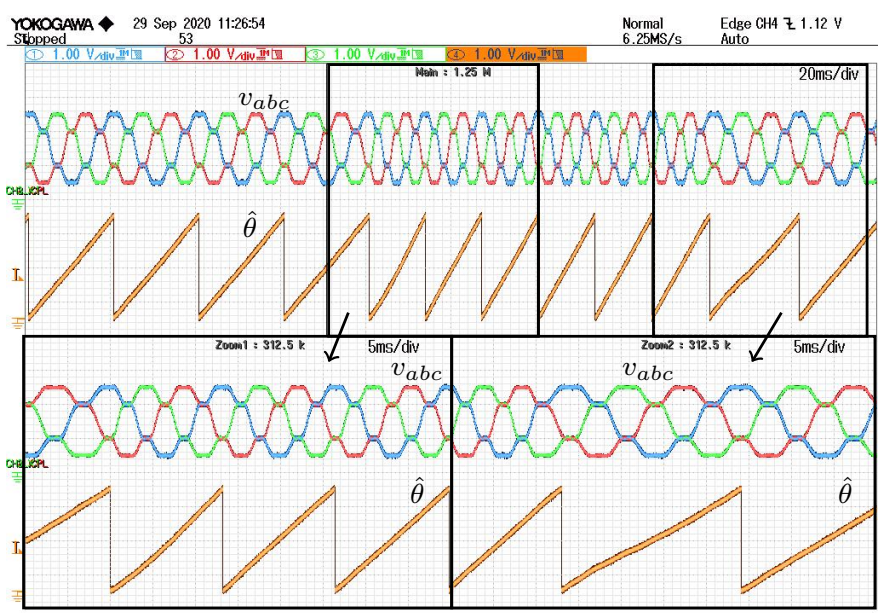

Fig. 16: Experimental results: behaviour of the CSMPLL-MCCF in the face of a frequency change from $50 \mathrm{~Hz}$ to $75 \mathrm{~Hz}$ for $60 \mathrm{~ms}$. Three-phase voltages, $v_{a b c}(t),(\mathrm{CH} 1, \mathrm{CH} 2, \mathrm{CH} 3)$ and the estimated phase $\hat{\theta}(\mathrm{CH} 4$, orange). Zoom 1 (bottom left): behaviour when the frequency ups to $75 \mathrm{~Hz}$. Zoom 2 (bottom right): behaviour when the frequency drops back to $50 \mathrm{~Hz}$.

iii) Easy implementation: the PLLs based on sliding modes are easily implementable by using simple sign functions.

iv) Thanks to i) and ii), the SMPLL and cSMPLL algorithms are interesting candidates for combining with pre-filtering stages (for example with the use of CCF and MCCF).

The proposed algorithms have been experimentally validated, including: the comparison with the traditional SRF-PLL and the use of the cSMPLL combined with the MCCF. Future works include the use of the proposed schemes for synchronization problems of power converters connected to the grid.

\section{REFERENCES}

[1] Y. Han, M. Luo, X. Zhao, J. M. Guerrero, and L. Xu, "Comparative performance evaluation of orthogonal-signal-generators-based single-

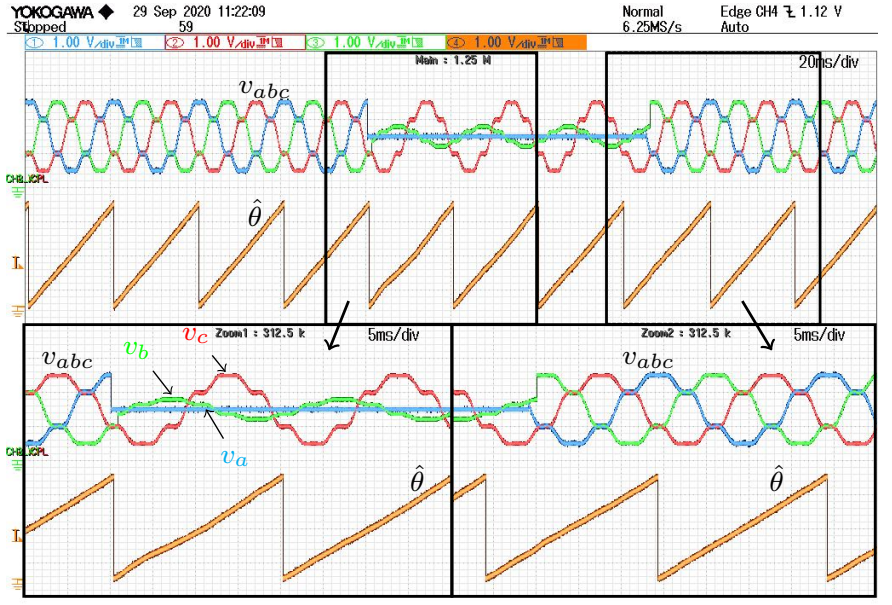

Fig. 17: Experimental results: behaviour of the cSMPLL-MCCF in the face of an unbalanced voltage sag for $60 \mathrm{~ms}$, where voltage amplitudes of phases $a$ and $b$ drop to $0 \mathrm{~V}$ and $0.25 \mathrm{~V}$. Three-phase voltages, $v_{a b c}(t),(\mathrm{CH} 1$, $\mathrm{CH} 2, \mathrm{CH} 3)$ and the estimated phase $\hat{\theta}(\mathrm{CH} 4$, orange). Zoom 1 (bottom left): behaviour when the voltage sag occurs. Zoom 2 (bottom right): behaviour when the voltages are recovered.

phase PLL algorithms - a survey," IEEE Trans. on Power Electronics, vol. 31 , no. 5, pp. 3932-3944, 2016.

[2] S. Golestan, J. M. Guerrero, and J. C. Vasquez, "Three-phase PLLs: A review of recent advances," IEEE Trans. on Power Electronics, vol. 32, no. 3, pp. 1894-1907, 2017.

[3] F. M. Gardner, Phaselock Techniques, 3rd ed. Wiley, 2005.

[4] B. Guo, S. Bacha, M. Alamir, A. Hably, and C. Boudinet, "Generalized integrator-extended state observer with applications to grid-connected converters in the presence of disturbances," IEEE Trans. on Control Systems Technology, vol. 29, no. 2, pp. 744-755, 2021.

[5] M. Karimi-Ghartemani and M. Iravani, "A nonlinear adaptive filter for on-line signal analysis in power systems: Applications," IEEE Trans. on Power Delivery, vol. 17, no. 2, pp. 617-622, 2002.

[6] M. Karimi-Ghartemani, "Linear and pseudolinear enhanced phasedlocked loop (EPLL) structures," IEEE Trans. on Industrial Electronics, vol. 61, no. 3, pp. 1464-1474, 2014.

[7] C. Buccella, C. Cecati, E. D. Santis, and H. A. Khalid, "Experimental verification of GEPLL architecture performance for grid connected inverter," in Proc. 42th Annual Conf. of the IEEE Industrial Electronics Society, 2016.

[8] S. Gude and C.-C. Chu, "Dynamic performance improvement of multiple delayed signal cancelation filters based three-phase enhanced-PLL," IEEE Trans. on Industry Applications, vol. 54, no. 5, pp. 5293-5305, 2018.

[9] D. S. S. Andrade, Y. N. Batista, F. A. S. Neves, and H. E. P. de Souza, "Fast phase angle jump estimation to improve the convergence time of the GDSC-PLL," IEEE Trans. on Industrial Electronics, vol. 67, no. 4, pp. 2852-2862, 2020.

[10] S. Golestan, M. Ramezani, J. Guerrero, F. Freijedo, and M. Monfared, "Moving average filter based phase-locked loops: Performance analysis and design guidelines," IEEE Trans. on Power Electronics, vol. 29, no. 6, pp. 2750-2763, 2014

[11] S. Reza, F. Sadeque, M. M. Hossain, A. M. Y. M. Ghias, and V. G. Agelidis, "Three-phase PLL for grid-connected power converters under both amplitude and phase unbalanced conditions," IEEE Trans. on Industrial Electronics, vol. 66, no. 11, pp. 8881-8891, 2019.

[12] H. A. Hamed and M. S. E. Moursi, "A new type-2 PLL based on unit delay phase angle error compensation during the frequency ramp," IEEE Trans. on Power Systems, vol. 34, no. 4, pp. 3289-3293, 2019.

[13] S. Golestan, J. M. Guerrero, and J. C. Vasquez, "DC-offset rejection in phase-locked loops: A novel approach," IEEE Trans. on Industrial Electronics, vol. 63, no. 8, pp. 4942-4945, 2016.

[14] Z. Dai, Z. Zhang, Y. Yang, F. Blaabjerg, X. Yao, and J. Zhang, "A luenberger observer-based phase locked loop for single-phase systems $t$ under harmonic disturbances," International Journal of Electrical Power and Energy Systems, vol. 116, p. 105528, 2020. 
[15] Y. Park, H.-S. Kim, and S.-K. Sul, "Frequency-adaptive observer to extract ac-coupled signals for grid synchronization," IEEE Trans. on Industry Applications, vol. 53, no. 1, pp. 273-282, 2017.

[16] W. Namgoong, "A modified proportional-integral loop filter to suppress DCO noise in digital PLL," IEEE Trans. on Circuits and Systems II, vol. 65 , no. 8, pp. 974-978, 2018.

[17] A. Bagheri, M. Mardaneh, A. Rajaei, and A. Rahideh, "Detection of grid voltage fundamental and harmonic components using Kalman filter and generalized averaging method," IEEE Trans. on Industrial Electronics, vol. 31, no. 2, pp. 1064-1073, 2016.

[18] H. Ahmed, S.-A. Amamra, and I. Salgado, "Fast estimation of phase and frequency for single-phase grid signal," IEEE Trans. on Industrial Electronics, vol. 66, no. 8, pp. 6408-6411, 2019.

[19] A. Verma, R. Jarial, P. Roncero-Sánchez, M. Ungarala, and J. Guerrero, "An improved hybrid prefiltered open-loop algorithm for three-phase grid synchronization," IEEE Trans. on Industrial Electronics, vol. 68, no. 3, pp. 2480-2490, 2021.

[20] A. Bolzoni and R. Perini, "Experimental validation of a novel angular estimator for synthetic inertia support under disturbed network conditions," in Proc. 21st European Conference on Power Electronics and Applications (EPE), 2019.

[21] S. Golestan, J. M. Guerrero, and J. C. Vasquez, "Modeling and stability assessment of single-phase grid synchronization techniques: Linear timeperiodic versus linear time-invariant frameworks," IEEE Trans. on Power Electronics, vol. 34, no. 1, pp. 20-27, 2019.

[22] S. Golestan, J. M. Guerrero, and G. B. Gharehpetian, "Five approaches to deal with problem of DC offset in phase-locked loop algorithms: Design considerations and performance evaluations," IEEE Trans. on Power Electronics, 2016.

[23] J. G. Rueda-Escobedo, S. Tang, and J. Schiffer, "A performance comparison of PLL implementations in low-inertia power systems using an observer-based framework," in Proc. IFAC World Congress, 2020.

[24] V. I. Utkin, J. Guldner, and J. Shi, Sliding Mode Control in ElectroMechanical Systems. CRC Press, 1999.

[25] V. F. Pires, G. D. Marques, and D. Sousa, "Phase-locked loop topology based on a synchronous reference frame and sliding mode approach for DVR applications," in Proc. International Conference on Computer as a Tool (EUROCON), 2011.

[26] M. Asad, A. I. Bhatti, and S. Iqbal, "Digital phase locked loop design using discrete time sliding mode loop filter," in Proc. IEEE 9th International Conference on Emerging Technologies (ICET), 2013.

[27] L. Harnefors, "Modeling of three-phase dynamic systems using complex transfer functions and transfer matrices," IEEE Trans. on Industrial Electronics, vol. 54, no. 4, pp. 2239-2248, 2007.

[28] A. Dòria-Cerezo, J. M. Olm, D. Biel, and E. Fossas, "Sliding modes of complex-valued nonlinear systems," IEEE Trans. on Automatic Control, in Early Access, 2021.

[29] X. Guo, W. Wu, and Z. Chen, "Multiple-complex coefficient-filter-based phase-locked loop and synchronization technique for three-phase gridinterfaced converters in distributed utility networks," IEEE Trans. on Industrial Electronics, vol. 58, no. 4, pp. 1194-1204, 2011.

[30] M. Ramezani, S. Golestan, S. Li, , and J. M. Guerrero, "A simple approach to enhance the performance of complex-coefficient filterbased PLL in grid-connected applications," IEEE Trans. on Industrial Electronics, vol. 65, no. 6, pp. 5081-5085, 2018.

[31] V. Kaura and V. Blasco, "Operation of a phase locked loop system under distorted utility conditions," IEEE Trans. on Industry Applications, vol. 33, no. 1, pp. 58-63, 1997.

[32] F. M. Serra, A. Dòria-Cerezo, and M. Bodson, "Multiple reference complex-based control for power converters under distorted and unbalanced conditions," IEEE Trans. on Power Electronics, (submitted).

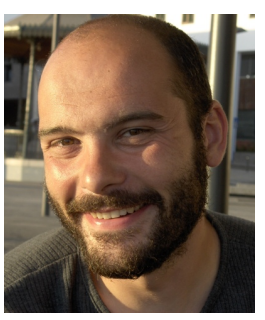

Arnau Dòria-Cerezo was born in Barcelona, Spain, in 1974. He received the undergraduate degree in electromechanical engineering and the Ph.D. degree in advanced automation and robotics from the Universitat Politècnica de Catalunya (UPC), Barcelona, Spain, in 2001 and 2006, respectively, and the DEA. degree in industrial automation from the Institut National des Sciences Appliquées de Lyon (INSALyon), Villeurbanne, France, in 2001.

Currently, he is an Associate Professor with the Department of Electrical Engineering, UPC. He carries out his research with the research group on Advanced Control of Energy Systems, Institute of Industrial and Control Engineering (IOC), UPC. From 2003 to 2004, he was a Control Training Site-Research Fellow with the Laboratoire des Signaux et Systèmes (L2S), Supélec, France. In 2010, he was a Visitor at the Technische Universiteit Delft, The Netherlands. His research interests include modeling and control of electrical systems and automotive applications.

He is an Associate Editor of the Control Engineering Practice since 2017.

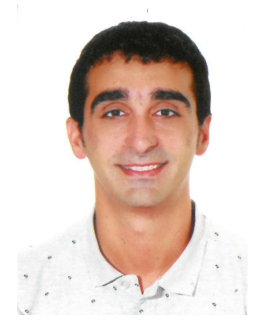

Víctor Repecho the B.S, M.S. and Ph.D. degrees in electronic engineering from the Universitat Politècnica de Catalunya (UPC), Barcelona, Spain, in 2006, 2012 and 2018, respectively. Since 2010, he has been a development engineer with the Institute of Industrial and Control Engineering (IOC) and since 2019 he has been an Assistant Professor in the Automatic Control Department, both at UPC. His research fields are related to digital control, nonlinear control, and control of power electronic converters.

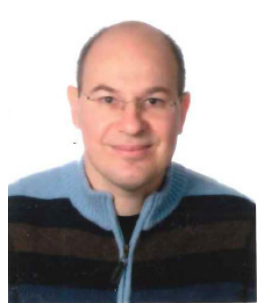

Domingo Biel received the B.S, M.S. and Ph.D. degrees in telecommunications engineering from the Universitat Politècnica de Catalunya (UPC), Barcelona, Spain, in 1990, 1994 and 1999, respectively. Since 1998, he has been an Associate Professor in the Electronic Engineering Department, UPC, where he teaches power electronics and control theory. $\mathrm{He}$ is the coauthor of around 25 papers in international journals and more than 70 communications in international conferences. His research fields are related to nonlinear control and its application to renewable energy systems and power electronics. 